\title{
MTADF : Multi hop Traffic Aware Data For WARDING FOR CONGESTION CONTROL IN WiRELESS SENSOR NETWORKS
}

\author{
Prabha $\mathrm{R}^{1}$, Prashanth Kumar Gouda ${ }^{1}$, Manjula $\mathrm{S} \mathrm{H}^{1}$, K R Venugopal ${ }^{1}$ and \\ L M Patnaik ${ }^{2}$ \\ ${ }^{1}$ Department of Computer Science and Engineering, University Visvesvaraya College \\ of Engineering ,Bangalore University, Bangalore, India \\ ${ }^{2}$ Honorary Professor, Indian Institute of Science, Bangalore 560 001, India
}

\begin{abstract}
In the past few years there is a remarkable change in the field of wireless sensor networks. Congestion occurs when there is a heavy traffic in the network. The heavy traffic in the network leads to wastage of energy and packet loss. Traffic Aware Dynamic Routing algorithm mitigates congestion by using one hop neighbor routing, hence throughput of the network is low. This paper proposed a Multi hop based Data Forwarding Technique to mitigate congestion. Queue length field and depth potential field play a major role to divert the traffic in the network to the alternate paths. The high traffic load leads to data queue overflow in the sensor nodes, these results in loss of important information about important events. Multi hop Traffic-Aware Dynamic Routing algorithm addresses congestion using depth potential field and queue length potential field. The algorithm forwards data packets around the congestion areas and scatters the excessive along multiple paths. The nodes with less load are efficiently utilized in response to congestion. The main aspect of the algorithm is to construct two independent potential fields using depth and queue length. Queue length field solves the traffic-aware problem. Depth field creates a backbone to forward packets to the sink. Both fields are combined to yield a hybrid potential field to make dynamic decision for data forwarding. Simulations are conducted to evaluate the performance of our proposed algorithm and our proposed scheme performs better compared to previous work.
\end{abstract}

\section{KEYWORDS}

Congestion Control; Depth Potential Field; Queue Length Potential Field; Traffic-Aware; Wireless Sensor Networks.

\section{INTRODUCTION}

Wireless sensors are equipped with a radio transceiver and a set of transducers through which they acquire information about the surrounding environment. When deployed in large quantities in a sensor field, these sensors can automatically organize themselves to form an ad hoc multihop network to communicate with each other and with one or more sink nodes that interact with a remote user. The user can inject commands into the sensor network via the sink to assign data collection, data processing and data transfer tasks to the sensors in order to receive the data sensed by the network. A diverse set of applications for sensor networks encompassing different fields have already emerged including medicine, agriculture, environment, military, inventory monitoring, intrusion detection, motion tracking, machine malfunction, toys and many others. 
Congestion occurs when the traffic load injected into the network exceeds available capacity at any point of the network. Congestion causes energy waste, throughput reduction, and increase in collisions and retransmissions at the Medium Access Control (MAC) layer. In addition, congestion results in the increase of queuing delays and information loss, leading to the deterioration of the offered Quality of Service (QoS), decrease of network lifetime and even the decomposition of network topology in multiple components. In traditional internet wired networks, buffer drops are taken as an indication of congestion while congestion control is usually carried out in an end-to-end manner (i.e. only the source-destination pair is involved) as opposed to the many-to-one nature of information transfer, which is a characteristic of sensor networks.

The congestion control mechanism in Wireless Sensor Networks (WSNs) is to be light and efficient at the same time. Congestion can appear in two main cases: the routers or intermediate device buffers are overflowed or channel collisions took place. Data packets sensed by sensor nodes can be classified into three types: clock-driven, event-driven and query-driven. Clockdriven data packets are gathered by the sensor nodes, and sent to the sink node periodically. In the emergency situation, the sensing data may be over the pre-set threshold value, the sensing node must send the event-driven data packets to sink node as soon as possible. In querydriven model, the sensor nodes are queried to reply data packets back to the sink. The eventdriven packet and query-driven packet have higher priorities to be delivered than the clockdriven data [1].

Congestion in WSNs degrades the performance of traffic flows present in the network. Possible causes of congestion in WSNs include: occurrence of a critical event, excessive event reporting, multimedia data and hot spots. The consequences of congestion are: decreased reliability, increased delay and jitter, and wastage of resources (i.e., bandwidth and energy). Therefore, without congestion detection and control mechanisms in place, meeting QoS requirements for inelastic applications becomes a daunting task.

\section{MOTIVATION}

Traffic-Aware Dynamic Routing to Alleviate Congestion in Wireless Sensor Networks (TADR) [2] work focused on alleviating the congestion in keeping view of fidelity level of the applications as the data generated during crisis state is more important. Whenever there is congestion in the network, congestion is handled by scattering the excessive packets to alternative paths with less load. The main drawbacks of TADR are more energy consumption and throughput is less due the one hop neighbour information used for congestion control.

\section{CONTRIBUTION}

The QoS parameters emphasized in this paper are throughput, delay and packet delivery ratio.

The forwarding node takes the load status of its neighbours into account to scatter the excessive packets. An appropriate alternate path consisting of idle or under loaded nodes is found based on load status of neighbours. Multi-Hop Traffic-Aware Dynamic routing paradigm, spreads the traffic in even patterns and resources are sufficiently utilized to reduce occurrence of congestion while improving overall throughput. The packets on the alternative route path will experience a relatively large end-to-end delay. This temporal spreading will benefit avoiding congestions appearing readily around the sink because of traffic centrality. The objective of our Multi-Hop 
Traffic-Aware Dynamic Routing (MTADF) is to alleviate congestion and improve throughput by distributing packets in both time and space. The main focus of this work is on detection and reduction of congestion in a particular node in WSNs. The proposed algorithm works based on two-hop routing technique. Due to heavy traffic in the network, the buffer size reaches threshold level which leads to congestion in the network. Multi-Hop based Traffic-Aware Data forwarding algorithm mitigates congestion by distributing the excessive packets through alternate paths. Thus idle or under loaded nodes are effectively utilized in response to congestion.

\section{RELATED WORK}

This section provides a brief overview of existing congestion control techniques. Congestion control in WSNs has gained high importance in the field of research. A detailed study on congestion control techniques reveals that the main goal of these techniques is to prevent the network from a congestive collapse. Which is a situation where in all the paths in the network are congested and the network is computing less useful work. Any transport protocol operating in the WSNs environment has two main components in its congestion control framework Congestion detection and Congestion avoidance. Congestion detection is crucial because if congestion detection is accurate, appropriate congestion control algorithms and techniques can be applied.

To design a routing protocol which saves sensor node energy while meeting the needs of different applications has become a research focus in wireless sensor network. Qingwei et al., [3] proposed Delay-Guaranteed Energy-Efficient Routing (DGEER) protocol, in which delay-sensitive packets are transmitted along the shortest path to minimize end-to-end delay. While others are sent to the next hop which is selected based on local neighbor information namely the remaining energy, the proportion covered by the length of data in the total length of the queue and the depth, which alleviates congestion at hot spots. The DGEER reduces the average end-to-end delay, balances the energy consumption, and prolongs network lifetime. Available congestion control schemes when applied to wireless networks results in packet drops and low throughputs and wastage of energy due to retransmissions. Maciej et al., [4] came up with a decentralized predictive congestion control for wireless sensor networks. This work consists of adaptive flow and adaptive backoff interval selection schemes devised with in consent with energy efficient distributed power control. The technique detects congestion using queue utilization and the embedded channel estimator algorithm which predicts the channel quality.

Jeongyeup et al., [5] discussed Rate Controlled Reliable Transport (RCRT) protocol suitable for constrained sensor nodes. RCRT uses end-to-end explicit loss recovery but places all the congestion detection and rate adaption functionality in the sinks. The two advantages of this protocol are efficiency and flexibility. Sumith et al., [6] came up with an idea of interference aware fair rate control technique which detects congestion at a node by monitoring the average queue length communicates congestion states to the set of potential interferers using a novel low overhead congestion sharing mechanism.

Shigang and Yang [7] described a congestion avoidance scheme based on lightweight buffer management. The author implemented simple yet effective approaches to prevent data packets from overflowing the buffer space of the intermediate sensors. The approach automatically adapts sensors forwarding rates to nearly optimal without causing congestion. Wang et al.,[8] implemented a novel upstream congestion control protocol for WSNs. The protocol is called as Priority based Congestion Control Protocol (PCCP). PCCP measures congestion as the ratio of packet inter-arrival time over packet-service time. PCCP achieves efficient congestion control and flexible weighted fairness. It achieves higher energy efficiency and better QoS in terms of both packet loss rate and delay. 
Raju et al., [9] addresses differentiated data delivery in the presence of congestion in wireless sensor networks. The basic protocol called Congestio Aware Routing discovers the congested zone of the network that exists between high priority data sources and the data sink using simple forwarding rules. It is not suitable for high mobile data sources. Jenn et al., [10] proposed a congestion control scheme that detects congestion using three steps i) Detecting long-term path congestions ii) Informing source of long-term path congestions iii)Reducing loading rate of source. To support quality of service requirements for multimedia applications having a reliable and fair transport protocol is necessary. Some applications of wireless multimedia sensor networks need to send real time traffic towards the sink node. The real time traffic requires low latency and high reliability. Mohammad et al., [11] distinguished high priority real time traffic from low priority non-real time traffic. A priority based rate control mechanism for congestion control and service differentiation in wireless multimedia sensor networks is presented.

Reza et al., [12] presented a lightweight congestion control mechanism. Capable of handling event-based and periodic upstream data flow towards the base station. The protocol functions in two phases, first phase uses ant based intelligence to find the shortest path. In the second phase protocol combines the knowledge of first phase and employs congestion control mechanism to reduce the packet loss.

\section{BACKGROUND}

The Wireless Sensor Networks have been implemented in many applications and has been the subject of many research activities recently. The major restrictions of the WSNs include limited energy supply, limited computing power, limited buffer size, and limited bandwidth. During emergency conditions, delivering data packets to the sink node as soon as possible is much more important than saving power. Network congestion happens when a link or node carries too much of data such that the network quality of service goes down. This in turn affects queuing delay and packet loss. Various techniques that exist in literature are limiting the sending rate, light weight buffer management, by passing the hotspot area, dynamic routing using bias, dynamic routing using steepest gradient method. Sensor networks usually operate under light load and become active in response to a detected or monitored event.

The energy constraint and the low buffer size are the two important problems in the sensor networks. It is hard to determine the sensing period required for a Sensor Node (SN) to sense and transmit data packets to the sink node in the clock-driven model. This is due to the fact that if the value is too short, the SN will produce and send data packets to the sink node more frequently. Consequently, the energy of the SN will be consumed more quickly. On the other hand, the WSNs cannot send the real time status to the sink node if the sensing period is too long. The low buffer size of a SN is an important problem in the design of the WSNs routing protocol. Most of the algorithms that route packets to destination via the shortest path, such as DestinationSequenced Distance Vector have tendency to saturate such shortest paths. Especially in multipriority applications, packets from all applications follow the same shortest path to the destination, making it congested and increasing the delays. In case of severe congestion, high priority packets always get through imposing either long delays or starvation for low priority applications. In the event driven scenarios, the events are intermittent and last for short time, therefore rate control mechanisms or feedback loop approaches do not perform well. Congestion will occur in a node when buffer is full, due to congestion some data will be dropped.

Congestion is a severe problem for wireless sensor networks. It causes the data to be retransmitted if the data is dropped. The data sensed by the sensor nodes is of two types: Event Driven Data and Periodic Data. Event driven data means the data which is generated on an occurrence of any particular event. For example the sensor monitoring the fire detection in forest 
will send data to sink immediately after the huge rise in temperature. Periodic data means data which is sent after equal amount of time interval. For example the sensor monitoring humidity of city will send data of humidity after every 6 hours to sink. The event driven nature leads to huge traffic in the network. Initially network is in idle load and suddenly become active when any event is detected, the generated data is of very high importance. Since all sensor nodes sends data, results in congestion in the network. This congestion problem arising in the network has great impact on quality of service parameter like energy, throughput, reliability and delay. So one must have to control congestion in WSNs because they deal with most of real time application where human life comes into picture.

\section{NETWORK MODEL}

In this section Network Model used and the Assumptions made are discussed along with preliminaries required for the design of our proposed MTADF Algorithm.

\subsection{Network Model and Assumptions Made}

The network is modelled as bowl structure as illustrated in Figure 1. The sink resides at the bottom and all data packets flow down along the surface just like water. When the traffic load in the network is light, the surface of the bowl is smoother and hence our algorithm acts just like the shortest path routing. In heavily loaded cases (e.g., burst of data packets caused by detection of a monitoring event), the congestion will form bulges on the bowl surface which blocks the packets to flow directly down to the bottom along the shortest path. The excessive packets are driven by the potential field to the appropriate alternative path without obstacles, i.e., idle or less loaded nodes. When the congestion disappears, the bowl surface becomes smoother, and the packets continue to move along the shortest path. Essentially, through spreading the packet transmissions spatially and temporarily, our MTDAF scheme alleviates congestion, while improving the throughput at the same time [2].

Table 1: Symbols used in the Algorithm

\begin{tabular}{l|l}
\hline Symbols & Meaning \\
\hline$N_{-} I D$ & Neighbour node ID \\
\hline$U_{-}$msg & Updated Message \\
\hline local depth & Depth value of the parent node \\
\hline$Q\left(N_{-} I D\right)$ & Queue length value of parent node \\
\hline$C$ & Normalized queue length of the neighbour node \\
\hline$D$ & $\begin{array}{l}\text { Cost of radio link between parent node and } \\
\text { neighbour node }\end{array}$ \\
\hline$Q$ & Depth of neighbour node \\
\hline$F_{d}\left(N_{-} I D\right)$ & Queue length of node \\
\hline$F_{q}\left(N_{-} I D\right)$ & $\begin{array}{l}\text { Depth force between parent node and neighbour } \\
\text { node }\end{array}$ \\
\hline$F_{m}\left(N_{-} I D\right)$ & $\begin{array}{l}\text { Queue length force between parent node and } \\
\text { neighbour node }\end{array}$ \\
\hline$A$ & $\begin{array}{l}\text { combined force between parent node and neighbour } \\
\text { node }\end{array}$ \\
\hline$P$ & Combined Co-efficient \\
\hline
\end{tabular}




\subsection{Preliminaries}

This section describes the construction of routing potential fields required for our proposed MTADF algorithm namely the depth field and the queue length field.

(i) Depth Potential Field: Provides the basic routing function (which the smooth bowl does), namely, to make each packet flow towards the sink. MTADF defines the depth potential field $V_{d}$ as $V_{d}=\operatorname{Depth}(v)$ where $\operatorname{Depth}(v)$ is the depth of node $v$. The depth field from node $v$ to its neighbour $w \in n b r(v)$ is given by

$$
F_{d}(v, w)=\left(V_{d}(v)-V_{d}(w)\right) / c_{v, w}
$$

$F_{d}(v, w)$ denotes the force between the parent node $v$ and the neighbour node $w$. This force is calculated by taking difference of depth field from parent node $v$ and its neighbour $w$ divided by cost of radio link between the node $v$ and neighbour $w$.

(ii) Queue Length Field: The priority routing function which determines the number of packets at each node in the network. In the bowl structure network model, packets move from a node to a neighbour with lower potential. To avoid a hotspot which is identified by a large queue, the potential at this node should be raised. Now, we define the queue length potential field.

$$
V q(v)=Q(v)
$$

$Q(v)$ denotes the normalized queue at node $v$ and it is defined as $Q(v)=$ Number of packets in the queue/Buffer size at node $v$.

\section{PROBLEM DEFINITION}

The objective of our MTADF algorithm is to alleviate congestion and improve throughput by distributing packets in both time and space. The main focus of this work is on detection and reduction of congestion in a particular node in WSNs. The algorithm works based on Twohop routing technique. Multi-hop based Traffic-Aware Data Forwarding for Congestion Control Algorithm forwards data packets around the congestion areas by spreading the excessive along multiple paths. The idle or under loaded nodes are efficiently utilized in response to congestion.

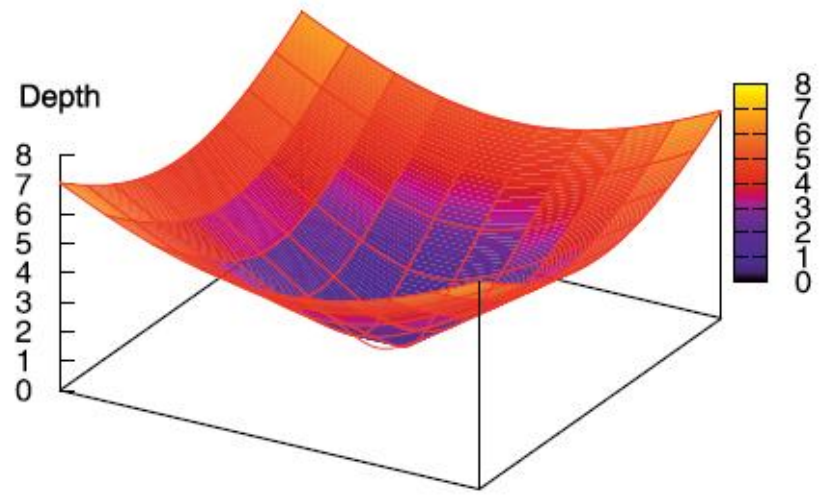

Figure 1: Bowl structure of the network 


\section{ALGORITHM}

This section explains the MTADF algorithm given in Table 2. MTADF needs the status information from nodes, such as queue length and depth, to construct the potential fields.

The WSNs is modelled as a bowl structure. In the bowl model each node will send data packets to the neighbours before, it reaches sink node. The data packets generated from source sensor nodes acts as input to the MTADF algorithm. The neighbour nodes receiving the data packets, finds the shortest paths to the sink by choosing the depth field and congestion free path using queue length potential field. The neighbours receiving the $N_{-} I D, U_{-} m s g$ from the neighbours, updates the parent node information into the routing table. The routing table contains the information like $S I N K_{-} I D, N_{-} I D, C, d, q, Q\left(N_{-} I D\right), F_{d}\left(N_{-} I D\right)$. Routing table contains information of all one hop neighbours and two hop neighbours routing information. Which includes $N \_I D, C, d, q, Q\left(N_{-} I D\right), F_{d}\left(N_{-} I D\right), F q\left(N_{-} I D\right), F_{m}\left(N_{-} I D\right)$ (Combined force field between parent and its neighbour).

Table 2: Multi-hop based Traffic Aware Congestion Control Algorithm (MTADF)

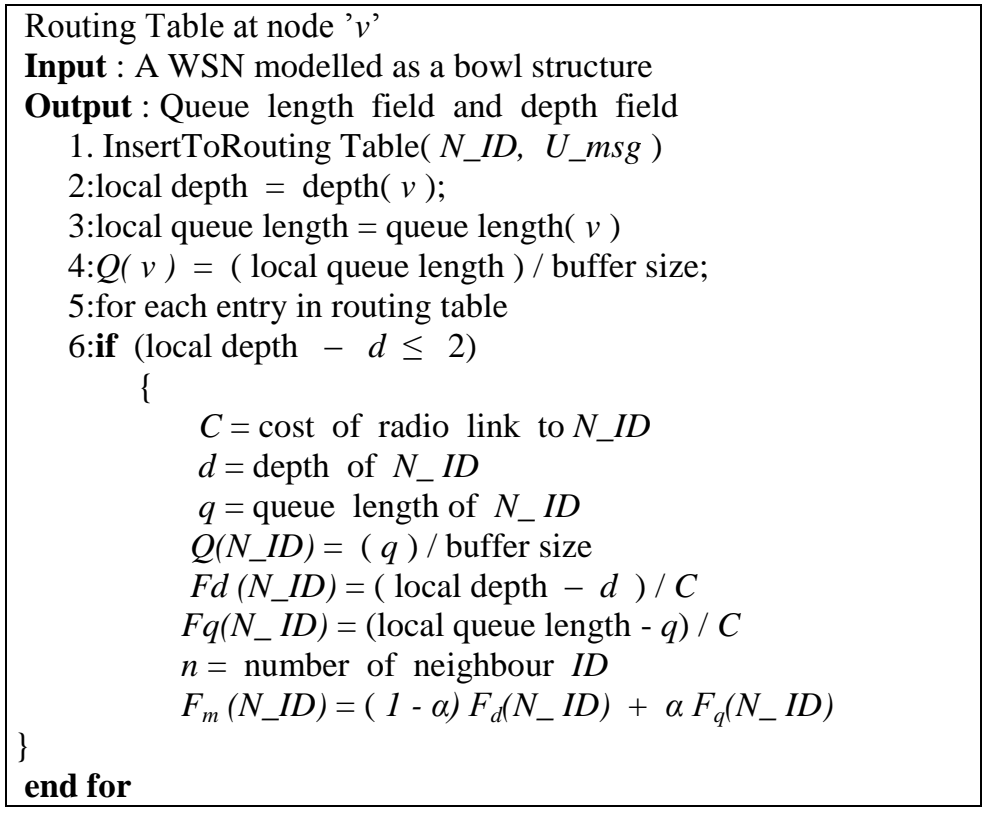

\section{PERFORMANCE EVALUATION}

This section details the simulation parameters used for the simulation of the MTADF algorithm and Performance analysis of the algorithm through the graphs. 


\subsection{Simulation Setup}

Table 3: Simulation Parameters

\begin{tabular}{l|l}
\hline Parameters & Value \\
\hline Area Size & $500 \mathrm{~m} * 500 \mathrm{~m}$ \\
\hline Number of nodes & $18-99$ \\
\hline Deployment Type & Random \\
\hline Transmission Range & $270 \mathrm{mtrs}$ \\
\hline Sink Coordination & $250 \mathrm{~m} * 250 \mathrm{~m}$ \\
\hline Initial Energy & $1 \mathrm{~J}$ \\
\hline $\begin{array}{l}\text { Link layer } \\
\text { transmission }\end{array}$ & $8 \mathrm{kbps}$ \\
\hline $\begin{array}{l}\text { Interface Queue } \\
\text { Type }\end{array}$ & Queue/DropTail/PriQueue \\
\hline Antenna & Antenna/OmniAnteena \\
\hline 0.1 seconds & Least Utilization Time \\
\hline 10 seconds & $\begin{array}{l}\text { Maximum Utilization } \\
\text { Time }\end{array}$ \\
\hline Application Type & Event Driven \\
\hline
\end{tabular}

The simulation parameters used in MTADF algorithm is shown in Table 3. The sensor nodes are first deployed randomly in square area with dimensions $500 \mathrm{~m} * 500 \mathrm{~m}$. The sink node is placed in the centre of the area. The WSN is modelled as bowl structure. The transmission range for all nodes is fixed for 270mts. The propagation model used is TwoRayGround. The MTADF algorithm is simulated under two scenarios: with high load in the network and with low load in the network

\subsection{Performance Analysis}

In this section, we evaluate the performance of MTADF algorithm using simulation experiments conducted on the NS2 [13] platform. NS2 uses OTcl and C++ codes to implement the given scenario. For a comprehensive performance evaluation, several QoS quantitative metrics considered are defined below.

(i) Energy Consumption: The average consumed energy per packet received by the sink reflects the energy efficiency of the protocols. It is ratio of the total energy consumption to the number of packets received by the sink successfully. The lower the energy consumed per packet, the higher the energy efficiency. 


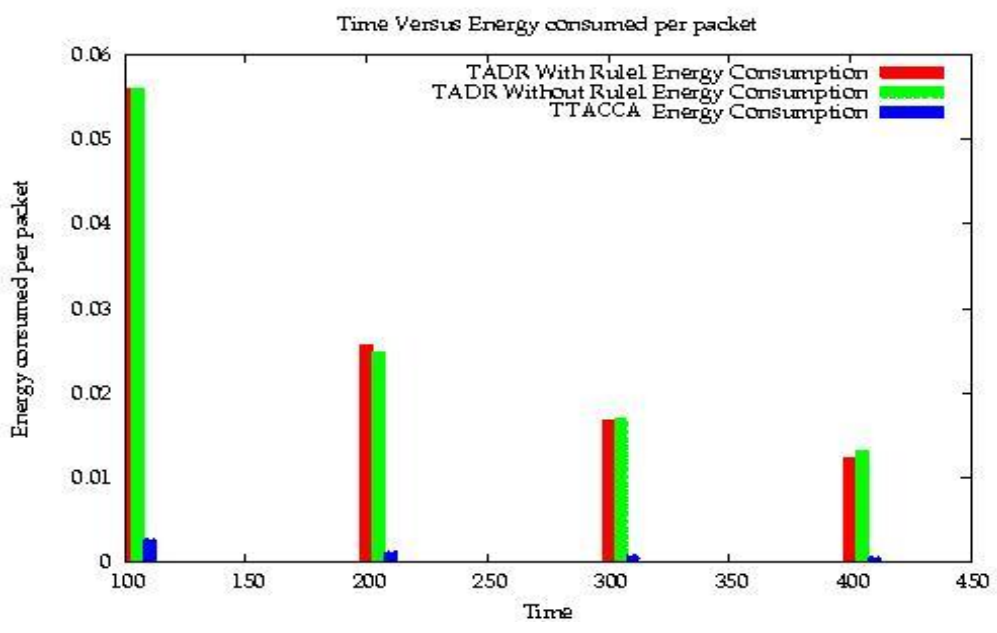

Figure 2: Time versus Throughput.

Figure 2 depicts the energy consumption of the MTADF algorithm. The energy consumed per packet is effectively reduced by $30 \%$ compared to the TADR algorithm. MTADF algorithm selects the forwarding node by considering distance to sink and forwarder node set information in the one hop neighbourhood or two hop neighbours. Considering distance to sink and forwarder node set information in the one hop neighbourhood or two hop neighbours. The queue length field in the one hop or two hop neighbour set solves the traffic aware problem. The depth filed gives the shortest path from parent node to the destination node. The number of hops travelled by the packet or message to reach the destination is comparatively less. Hence the energy consumed for each packet to reach the destination or sink node is less.

(ii) Receiving Packets Ratio (RPR): It is defined as the rate at which the sink receives packets. It is the appropriate metric that reflects the effect of caching and spreading over the time and space because RPR will keep a nonzero value for a relatively long period if the excessive packet is spread spatially and temporarily.

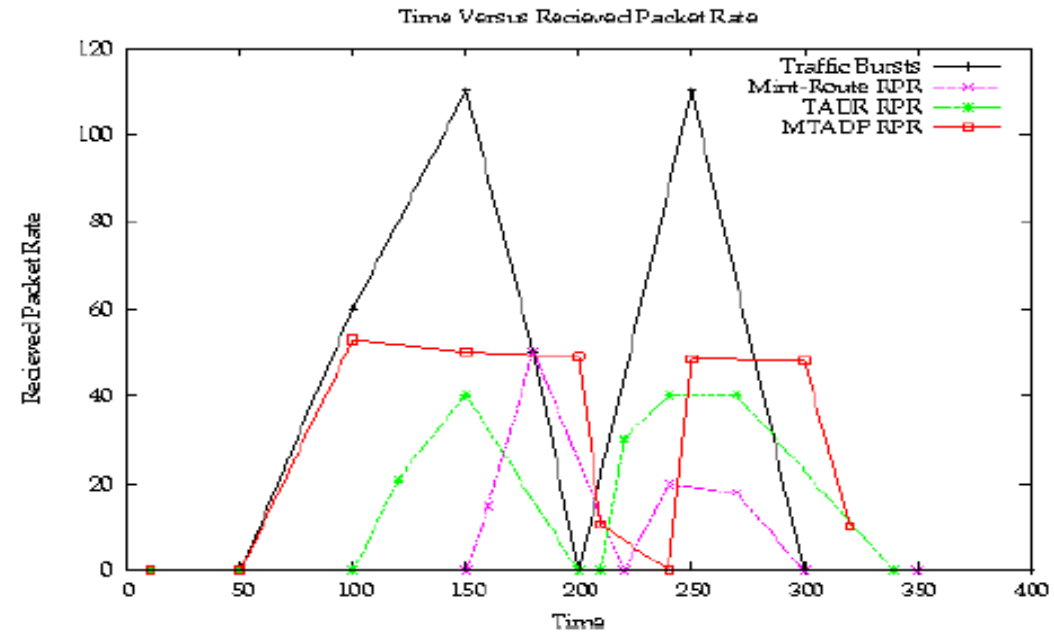

Figure 3: Time Versus Received Packet Ratio. 
Figure 3 shows the comparison of time versus received packet rate. As shown in the figure the network burst represents high load in the network. In TADR there are 110 packets out of which TADR receives only 48 packets at sink node. Hence the RPR of TADR is $48 \%$. MTADF forwards the packets using two hop transmission due to this 53 packets are received at the sink. Hence RPR of MTADF is 53\%. In MTADF at simulation time equal to 55 seconds, it receives 53 packets out of 110 packets and continues to receive 53 .

(iii) Delay: The time taken between the source node sending the packet and the destination node receiving the packet is End-to-End Delay. The average of the End-to-End delay of all the packets transmitted between each of the pairs of source-destinations gives the average End-to-End delay.

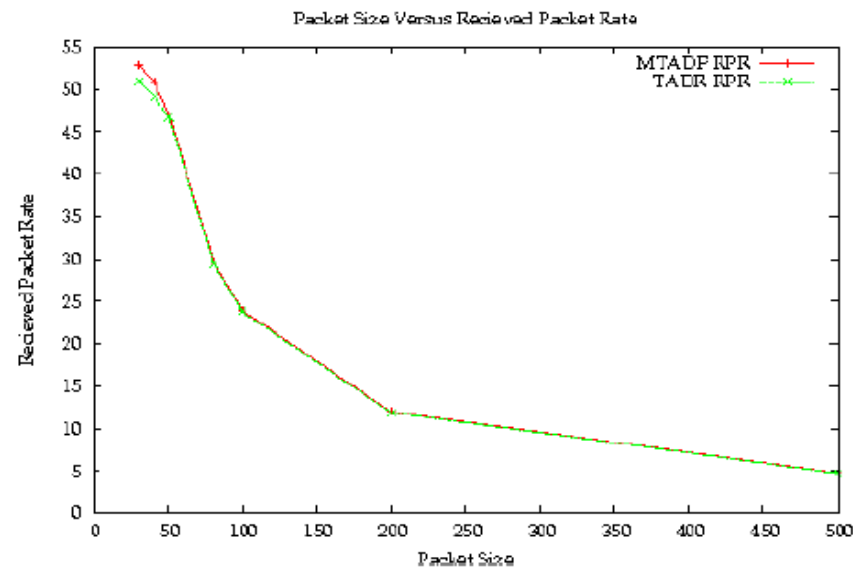

Figure 4: Packet Size versus Received Packet Rate

Figure 4 shows the comparison of packet size versus received packet rate. In TADR packet is forwarded by one of routing technique so that receiving packet rate at sink node is comparatively low. Whereas in our work MTADF the packet is forwarded to the destination through two hop routing technique and one hop routing technique if there is no congestion in the network. Hence the receiving packet rate of MTADF is comparatively high.

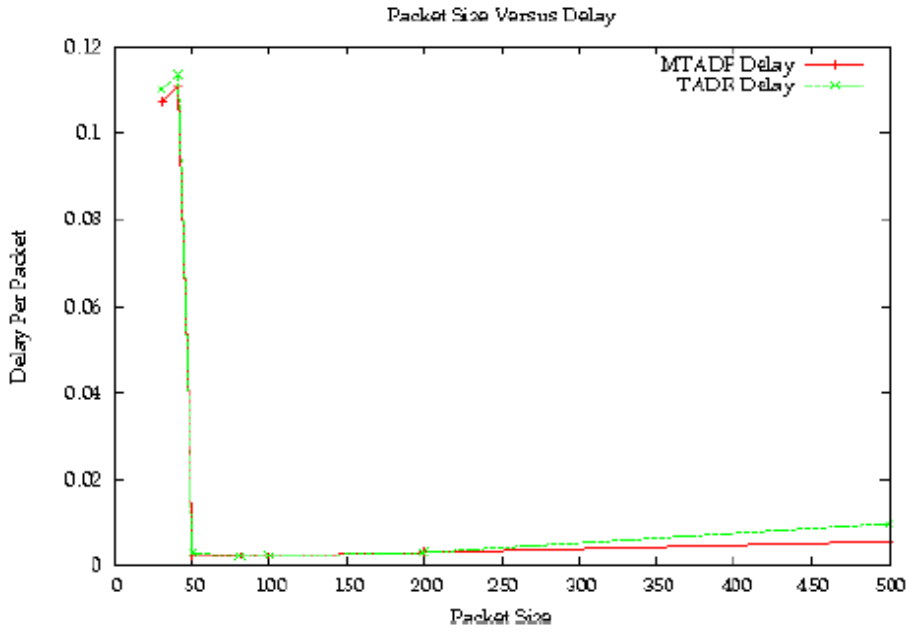

Figure 5: Time versus Delay per Packet

Figure 5 shows the comparison of packet size versus delay. When the packet size is low ( 25 bytes to 50 bytes) the frequency of packet generated gets increased. Thus creating high load in the network. Hence there will be packet dropping. TADR routes the packets to multiple hops before it 
reaches the sink. Hence the delay is comparatively high. Whereas MTADF routes the packets by considering two hop neighboring nodes, before the packet reaches the destination. Due to the two hop transmission the delay incurred is comparatively low. As packet size reaches 50 bytes and above the delay will drastically declined to low value. For packet size 50 and above the delay is low due to the light traffic in the network.

\section{CONCLUSIONS}

The congestion control in WSNs is different from that in tradition networks, such as Wireless LAN and adhoc networks. The pure traffic control is able to alleviate congestion, but hard to satisfy the fidelity required by applications. In this paper, MTADF algorithms follows the philosophy of dynamic capacity planning to deal with the congestion problem in WSNs. Through MTADF algorithm QoS performance metrics delay is reduced by $10 \%$, improves energy efficiency by $30 \%$ and throughput of MTADF is high compared with TADR. The MTADF algorithm thus mitigates congestion in the network efficiently compared to TADR congestion control algorithm.

\section{REFERENCES}

[1] Mansoor-uz-Zafar Dawood, Biztek, Pakistan,Noor Zaman, Abdul Raouf Khan, Mohammad Salih,'Designing of Energy Aware Quality of Service (QoS) Based Routing Protocol for Efficiency Improvement in Wireless Sensor Network", Journal of Information and Communication Technology, vol. 4, no. 1, 2010.

[2] Fengyuan Ren, Tao He, Sajal K. Das and Chuang Lin, "Traffic-Aware Dynamic Routing to Alleviate Congestion in Wireless Sensor Networks", IEEE Transactions On Parallel and Distributed Systems, vol. 22, no. 9, September 2011.

[3] Qingwei Liang, Daoyuan Yao, Sui Deng, Siliang Gong, and Jian Zhu, "Potential Field Based Routing to Support QoS in WSN", Journal of Computational Information Systems, 2012

[4] Maciej Zawodniok and Sarangapani Jagannathan,"Predictive Congestion Control Protocol for Wireless Sensor Networks", IEEE Transactions on Wireless Communications, vol. 6, no. 11, November 2007.

[5] Jeongyeup Paek and Ramesh Govindan, "RCRT: Rate Controlled Reliable Transport for Wireless Sensor Networks.”,SenSys, 2007

[6] Sumit Rangwala, Ramakrishna Gummadi, Ramesh Govindan and Konstantinos Psounis," Interference Aware Fare Rate Control in Wireless Sensor Networks", SigComm, 2006.

[7] Shigang Chen and Na Yang, "Congestion Avoidance based on Lightweight Buffer Management in Sensor Networks.” Systems IEEE Transactions on Parallel and Distributed, vol. 17, no. 9, September 2006.

[8] C Wang, B Li, K Sohraby, and M Daneshmand, "Upstream Congestion Control in Wireless Sensor Networks Through Cross-Layer Optimization.” IEEE Journal on Selected Areas in Communications, vol. 35, no. 4, May 2007.

[9] Rajkumar, Riccardo Crepandi, Hosam Rowaihy, Albert F Harris, Guohong Cao and Thomas F Laporta, "Mitigating Performance Degradation in Congested Sensor Networks.", IEEE Transactions on Mobile Computing, vol. 7, no. 6, July 2008.

[10] Jenn-Yue Teo, Yajun Ha and Chen-Khong Tham, 'Interference -Minimized Multipath Routing with Congestion Control in Wireless Sensor Networks for High-Rate Streaming", IEEE Transactions on Mobile Computing, vol. 7, no. 9, July 2008.

[11] Mohammad Hossen, Yaghmaee and Donald A Adjeroh, "Priority-based Rate Control for Service Differentiation and Congestion Control in Wireless Multimedia Sensor Networks", Computer Networks, 2009.

[12] Reza Ghasem Aghaei, Mahfujur Rahman, Md Abdur Rahman, Wail Gueaieb and Abdulmotaleb Saddik,"Ant Colony-Based Many-to-One Sensory Data Routing in Wireless Sensor Networks", IEEE, 2008.

[13] http://www.isi.edu/nsmam/ns 


\section{Authors}

Prabha $\mathbf{R}$ is currently working as an Associate Professor in the Department of Information Science and Engineering, Dr. Ambedkar Institute of Technology, Bangalore, India. She obtained her Bachelor of Engineering degree in Computer Science and Engineering branch. M.E in Computer Science and Engineering from Computer Science Department, UVCE, Bangalore University in the year 2003. She has 22 years of teaching experience. Currently she is pursuing Ph. D in the Department of Computer Science and Engineering, University Visvesvaraya College of Engineering, Bangalore University, Bangalore. Her research interest is in the area of Wireless Sensor Networks.

Prashanth Kumar Gouda received his B.E in Electronics and Communication Engineering from Dr. Ambedkar Institute of Technology Bangalore from Visvesvaraya Technological University in the year of 2010. M.E in Computer Networks from Computer Science Department, UVCE, Bangalore University in the year 2013. His research focus includes QoS and Routing in WSN and Cloud Networking and Communication. He worked as Business Intelligence Developer at GrayMatter Software Service Private limited Bangalore.

Dr. S H Manjula is currently working as an Associate Professor in the Department of Computer Science and Engineering, University Visvesvaraya College of Engineering Bangalore University, Bangalore, India. She obtained her Bachelor of Engineering degree in Computer Science and Engineering branch, Masters of Engineering and $\mathrm{Ph} \mathrm{D}$. in Computer Science and Engineering. She has published a book on Wireless Sensor Networks. She has published more than 50 papers in refereed international journals and conferences. Her research interests are in the field of Wireless Sensor Networks, Semantic web and Data Mining.

Dr. Venugopal K R is currently Special Officer, DVG Bangalore University and Principal, University Visveswaraya College of Engineering, Bangalore University Bangalore. He obtained his Bachelor of Engineering from University Visvesvaraya College of Engineering. He received his Master's degree in Computer Science and Automation from Indian Institute of Science Bangalore. He was awarded Ph. D in Economics from Bangalore University and Ph.D in Computer Science from Indian Institute of Technology, Madras. He has a distinguished academic career and has degrees in Electronics, Economics, Law, Business Finance, Public Relations, Communications, Industrial Relations, Computer Science and Journalism. He has authored and edited 39 books on Computer Science and Economics, which include Petrodollar and the World Economy, C Aptitude, Mastering C, Microprocessor Programming, Mastering C++ and Digital Circuits and Systems. During his three decades of service at UVCE he has over 400 research papers to his credit. He was a Post-Doctoral Research Scholar at University of Southern California, USA. His research interests include Computer Networks, Wireless Sensor Networks, Parallel and Distributed Systems, Digital Signal Processing and Data Mining.

Dr L M Patnaik is honorary professor in Department of Computer Science and Automation, Indian institute of Science, Bangalore. During the past 35 years of his service at the Institute he has over 700 research publications in refereed International Conference Proceedings. He is a Fellow of all the four leading Science and Engineering Academies in India. Fellow of the IEEE and the Academy of Science for the developing world. He has received twenty national and international awards. Notable among them is the IEEE Technical Achievement Award for his significant contributions to High performance Computing and Soft Computing. He is an Ex-Vice Chancellor Defence institute of Advanced Technology, Pune India. His area of research interest has been Parallel and Distributed Computing, Mobile Computing, CAD for VLSI circuits, Soft computing and Computational Neuroscience. 